\title{
Modeliser la realite materielle dans l'enseignement des mathematiques
}

Eric Laguerre

\section{Résumé}

Notre problématique consiste à tenter de définir un acte de modélisation du réel, qui sera considéré comme matériel tout au long de notre travail, et à illustrer cet acte en prenant appui sur un problème en situation élaboré dans le but, du point de vue des étudiants, d'une part, de revisiter la trigonométrie, d'autre part, de trouver une solution à une problématique professionnelle liée à la mesure de distances inaccessibles.

Mots-clés: enseignement, trigonométrie, modélisation, problème en situation, distances inaccessibles

\begin{abstract}
Our problematics is to try to define a act act of modelizing reality, that will be considered as concrete throughout our work. We will illustrate this act by analyzing a situated problem which has been especially built in a twofold prospect : firstly for students, in order to revisit trigonometry, secondly, to find a solution to a professional problem related to measuring inaccessible distances.
\end{abstract}

Keywords: teaching, trigonometry, modeling, situated problem, inaccessible distances 


\section{Introduction}

Notre travail consiste à nous intéresser aux mécanismes de la mathématisation de la réalité matérielle dans l'enseignement au sein de la formation initiale dispensée dans le cadre de l'Ecole Nationale des Techniciens de l'Equipement dont nous avons été élève de 1986 à 1987 et au sein de laquelle nous sommes ponctuellement intervenus en tant qu'enseignant quelques années plus tard dans le cadre d'une expérimentation. Les techniciens supérieurs de cette école participent aux phases d'études de travaux et de contrôle dans le domaine de l'aménagement, de l'urbanisme, de la construction, de l'entretien et de la modernisation des routes, des ponts, des ports, des aéroports et des voies navigables. Ils produisent des plans à l'aide de la DAO1 en entrant manuellement des données ou en les transférant directement de topomètres. A cet effet, ils réalisent à l'aide d'appareils optiques des mesures de distances, de dénivellations, ils étudient la nature du sol et délimitent aussi les terrains.

Notre problématique consiste à tenter de définir un acte de modélisation du réel, qui sera considéré comme matériel tout au long de notre travail, et à illustrer cet acte en prenant appui sur un problème en situation élaboré dans le but, du point de vue des étudiants, d'une part, de revisiter la trigonométrie, d'autre part, de trouver une solution à une problématique professionnelle liée à la mesure de distances inaccessibles.

Dans un premier temps, en nous fondant sur des travaux déjà entrepris par d'autres chercheurs, nous définissons tout d'abord la modélisation du réel dans le cadre de l'enseignement puis ce que peut représenter le concept de réalité matérielle. Nous complétons et adaptons ces définitions en proposant deux approches du sens de la réalité et de l'acte de modélisation du réel qui gagnent à être peaufinées grâce à une analyse sémiotique plus fine.

Dans un second temps, nous élaborons un problème en situation. Nous pouvons dire dès à présent qu'un problème en situation ${ }^{2}$, qui n'est pas une situation problème, est expérimenté de façon effective par les apprenants. Cette expérimentation n'a pas nécessairement lieu dans la réalité du monde physique qui nous entoure mais éventuellement au sein d'une représentation ou d'une modélisation de ce dernier.

\section{Problématique}

Depuis bien longtempsles scientifiques et les philosophes s'interrogent sur les rapports qui peuvent être établis entre les mathématiques et la réalité en particulier par le biais de la mathématisation du réel. La mathématisation de la réalité prend une forme toute particulière depuis quelques décennies.

\footnotetext{
${ }^{1}$ Dessin Assisté par Ordinateur

${ }^{2}$ Gravemeijer \& Doorman, « Context problems in realistic mathematics education.... »
} 
Même si aucune scission n'est envisagée entre les deux démarches notre problématique ne consiste pas à nous questionner sur la modélisation mathématique théorique de la réalité matérielle mais sur la mathématisation du réel dans l'enseignement. Nous émettons l'hypothèse que la construction d'une modélisation de la réalité de la part des étudiants dans le cadre des mathématiques enseignées a un intérêt du point de vue mathématique mais également du point de vue de l'appréhension que chacun peut avoir du monde qui l'entoure. Nous pouvons d'ores et déjà dire que d'une manière générale, notre recherche consiste à tenter de clarifier les deux concepts de réalité matérielle et de modélisation en situation d'enseignement. Les trois questions de notre problématique sont les suivantes:

- Qu'est-ce que la réalité matérielle dans l'enseignement des mathématiques ?

- En quoi peut consister la modélisation de la réalité matérielle dans l'enseignement des mathématiques?

- Comment mener à bien une telle modélisation avec des étudiants ?

\section{Cadres théoriques}

Pour répondre à ces trois questions, nous avons décidé d'adopter une approche à la fois didactique et philosophique.

Nous allons tout d'abord tenter de cerner le concept de modèle au sens large du terme. Puis nous allons nous intéresser à un cadre théorique plus spécifiquement didactique qui prenne simultanément en compte la dimension de l'étudiant, de l'enseignant et d'un contexte externe.

A partir du constat de certaines insuffisances que nous aurons constatées au sein d'un cadre théorique didactique au sujet précisément du concept de réalité matérielle, nous le compléterons par une approche de la réalité afin d'affiner la notion de modélisation.

Nous terminerons notre travail par l'élaboration et l'analyse d'un problème en situation qui illustrera l'acte de modélisation du réel dans l'enseignement et qui nous permettra in fine de peaufiner notre approche.

\subsection{Les types de modèles de Paul Ricœur}

Même si nous nous intéressons plus spécifiquement à la modélisation dans l'enseignement des mathématiques, l'approche du concept de modèle en science de Paul Ricœur nous semble tout à fait éclairante pour notre sujet : 
"Dans le langage scientifique, le modèle est essentiellement un instrument heuristique qui vise, par le moyen de la fiction, à briser une interprétation inadéquate et à frayer une interprétation nouvelle plus adéquate » ${ }^{1}$.

Dans son ouvrage, l'auteur distingue trois types de modèles. Le premier type est qualifié de "modèle à l'échelle " qui est une représentation miniaturisée ou agrandie de l'objet en question qui permet par exemple de mettre en évidence des caractéristiques invisibles jusque là. Nous retrouvons ce type de modèle dans de nombreuses études d'aérodynamique entreprises en soufflerie à partir de représentations à l'échelle d'aéroplanes ou également au sein des travaux de recherches étudiant en laboratoire le mouvement de la houle ou les conditions de déclanchement des avalanches à partir de reproductions fidèles du milieu naturel de chaque situation.

Un second type de modèle est qualifié par l'auteur de " modèle analogique " pour lequel un phénomène est comparé par similitude à un autre phénomène. Par exemple, un circuit électrique peut avoir des analogies avec un circuit hydraulique. L'étude de la chute du sable d'un sablier peut être associée à la chute progressive d'une chaîne le long d'une paroi dans le but de mieux prendre en considération le fait que, dans les deux cas mais pour des raisons différentes, la masse en mouvement varie avec le temps.

Le troisième type de modèle est nommé "modèle théorique ". Ce type de modèle nécessite l'utilisation d'un langage nouveau qui favorise l'apparition de nouvelles appréhensions de l'objet étudié. Paul Ricœur prend comme exemple de l'élaboration possible d'un modèle l'activité des enzymes, ces dernières étant représentées par des formes géométriques précises comme des carrés et des cercles. Nous aurons l'occasion d'illustrer plus amplement cette catégorie de modèle lors de l'étude de notre situation d'enseignement avec des lorgnettes.

Sauf erreur de notre part, ces types de modèles n'ont pas été investis dans le cadre d'un enseignement prenant en compte à la fois les dimensions "élève " et " professeur ". Compte tenu de notre problématique, il nous semble important de nous fonder sur un cadre théorique en rapport avec l'enseignement et qui tienne compte des objectifs des enseignants et des conceptions des étudiants. La didactique des domaines d'expériences ${ }^{2}$ semble intéressante à ce sujet. Les types de modèles décrits précédemment et élaborés par Paul Ricœur constitueront un complément ponctuel d'analyse de notre situation d'enseignement.

\footnotetext{
${ }^{1}$ Ricœur, De l'interprétation. Essai sur Freud.

2 Boero, « Teaching and Learning Geometry in Contexts... ».
} 


\subsection{La didactique des domaines d'expérience}

Le cadre théorique de référence des DDE est, d'une part, celui de Zone Proximale de Développement(ZPD) ${ }^{1}$ et, d'autre part, celui de la dialectique outil/objet ${ }^{2}$ et de la transposition didactique $^{3}$. Dans laZPD, à partir d'une connaissance mathématique donnée, il s'agit de prendre en compte l'écart qui existe entre ce qu'est capable de résoudre un sujet seul et ce dont il est capable avec l'aide d'une personne après apprentissage. En ce qui concerne la dialectique outil/objet, un concept mathématique a deux statuts différents : le statut outil conceptualisé par la résolution de problèmes et le statut objet en tant qu'objet d'étude dans l'édifice des mathématiques. Et enfin, pour ce qui est de la transposition didactique, nous nous référons aux transformations que subit un savoir savant pour le transformer en savoir scolaire. II s'agit du passage d'un savoir de production (recherche) à un savoir qui vit dans un espace de communication, de diffusion (école). Nous pouvons maintenant développer le cadre de la DDE qui est relatif aux relations qui se développent à l'école entre :

- le contexte interne de l'élève - ses conceptions, l'ensemble de ses schèmes, représentations, émotions, analyse -, qui lui permet de faire un repérage des données du monde réel, un choix d'hypothèses supplémentaires sur le monde réel si cela est nécessaire ce qui aboutit à un choix de représentation du monde réel

- le contexte interne de l'enseignant - ses conceptions, ses objectifs d'apprentissage, représentation, émotion, attentes -

- et le contexte externe - portion de réalité, objet de réflexion, signes, objets, contraintes physiques, discussion, analyse. Dans notre situation, c'est à partir de diverses représentations du réel que des discussions s'engagerons pour en retenir une peut être plus pertinente que les autres.

Dans ce cadre, un modèle est construit collectivement par les élèves qui se fondent sur diverses versions de représentations de la situation qui correspondent à autant de contextes internes des élèves. Ces modèles sont construits en utilisant des artefacts matériels (supports, matériaux, objets, signes, ordinateur...), nous nous trouvons alors au niveau des modèles informels, et des artefacts formels liés aux mathématiques (parallèles, rationnels...) et aux registres symboliques qui font émerger des modèles formels.

Un modèle peut être également employé en tant qu'artefact et donc en tant que nouvelle réalité pour réaliser un nouveau modèle. La modélisation est alors caractérisée par la dialectique comprenant diverses étapes d'interprétation qui peut être établie entre une situation observée et

\footnotetext{
${ }^{1}$ Vygotski, Pensée et langage.

${ }^{2}$ Douady, "Jeux de cadres et dialectique outil-objet ».

${ }^{3}$ Chevallard, La transposition didactique....
} 
ses représentations correspondant à différents modèles ${ }^{1}$.

La DDE a par ailleurs été utilisé par Nadia Douek au sein d'une expérimentation en rapport avec les conceptions que peuvent avoir des élèves de l'école primaire au sujet de la position du soleil et de la longueur de l'ombre d'une personne. Par exemple, pour Stefano : " plus le soleil est haut plus l'ombre est longue » et pour Steven : " plus le soleil est loin plus l'ombre est longue $"$.

En résumé, dans le cadre théorique des $\mathrm{DDE}$, nous pouvons dire que les modèles sont élaborés matériellement et en prenant appui également sur des représentations sémiotiques. L'acte de modélisation se définit par le discours qui accompagne l'élaboration et la description des modèles. En ce qui concerne le contexte externe qui apparaît dans ce cadre théorique, les signes, les contraintes physiques, les discussions et l'analyse semblent assez clairement définis. Mais qu'en est-il de la réalité ou des portions de réalité ? II est légitime de se poser la question suivante : de quelle réalité parlerons-nous ? Ce sens n'est pas encore clarifié que ce soit dans le cadre de la DDE où dans d'autres cadres de la didactiques dans lesquels est posée cette question de la modélisation. Comme nous cherchons à définir ce que pourrait être la modélisation du réel dans l'enseignement des mathématiques, il nous semble important que notre réflexion porte avant tout sur ce que nous pouvons entendre derrière le vocable " réalité matérielle».

\subsection{Monde réel et modélisation de la réalité}

La réalité n'est pas toujours un objet matériel ou un phénomène naturel ou social mais elle peut être représentée sous une forme en partie modélisée ${ }^{2}$. L'idée qui est à prendre en considération est le fait qu'un modèle apparaissant à un certain niveau de la modélisation du réel constitue une nouvelle réalité ce qui correspond à la prise en compte de l'existence de métaniveaux d'interprétations de la réalité. Mais cette dernière n'est toujours pas clairement définie c'est la raison pour laquelle nous nous tournons du côté de la philosophie. Dans son ouvrage, Thirion $^{3}$ citeC. Rosset qui conçoit la réalité comme un certain niveau de notre rapport au monde en tant qu'il est envisagé sous les catégories de l'événement et de la situation. Nous retenons cette approche mais afin qu'il n'y ait pas de confusion possible entre le terme de situation tel qu'il est entendu par le philosophe et d'autres acceptions possibles de ce vocable nous le remplaçons par le mot contexte même si ce dernier est également polysémique.

\footnotetext{
${ }^{1}$ Douek, « Argumentation and conceptualization in context.... », p. 91.
}

2 Dapueto \& Parenti, «Contributions and obstacles of contexts in the developpement of mathematical knowledge ».

${ }^{3}$ Thirion, Les mathématiques et le réel. 
Nous entendons par événement la donnée d'un espace, d'un temps et de faits ou plutôt d'une interprétation de faits.

Nous comprenons le vocable " contexte » en tant que ce dernier est caractérisé par un environnement, un matériel et des actions. Au premier abord, nous appelons modélisation la dialectique établie entre un contexte et les événements observés et leurs représentations respectives à travers diverses étapes qui correspondent à autant de modèles. Ces modèles sont construits en utilisant des artefacts matériels (supports, matériaux, objets, signes, ordinateur...), nous nous trouvons alors au niveau des modèles informels, et des artefacts formels liés aux mathématiques (parallèles, rationnels...) qui font émerger des modèles formels. Pour être adaptés à des situations d'apprentissage, nous pouvons considérer que les modèles doivent remplir deux conditions $^{1}$ : ils naissent dans des contextes qui peuvent être perceptibles, c'est-à-dire des contextes pour lesquels tous les éléments sont visibles et palpables, ou dans des contextes qui peuvent être imaginables, c'est-à-dire qu'à l'inverse certaines parties de ces modèles sont à imaginer et ne sont pas visibles dans la réalité initiale qui est modélisée. Nous illustrerons cela ultérieurement en prenant appui sur notre situation d'apprentissage.

A partir d'un contexte tiré de la réalité et d'événements liés à ce dernier, un déplacement s'effectue de modèle à modèle pour créer une nouvelle interprétation de la réalité initiale ou une nouvelle réalité. A priori, nous pouvons dire qu'il y a modélisation lorsque nous passons d'un cadre interprétatif strictement verbal d'un fait " concret » détaché de toute autre référence que le langage commun, à un autre cadre interprétatif par l'entremise d'artefacts qui servent de médiateurs dans le but de faire émerger une nouvelle compréhension de l'événement ou l'apparition de nouveaux événements. Nous proposons de partir de ce sens général de la « modélisation du réel » en tant que pratique d'enseignement en ayant maintenant pour objectif de l'affiner à la lumière de la situation qui suit et qui prend appui sur les relations qui peuvent être établies entre le macro-espace, le méso-espace et le micro-espace ${ }^{2}$. Le macro-espace est l'espace pour lequel, par exemple, la mesure de longueurs n'est pas possible à l'aide des instruments de la géométrie. Dans le méso-espace, ces mesures sont réalisables par des méthodes d'arpentage. Alors que dans le micro-espace, ces mesures peuvent être effectuées directement à l'aide des instruments de la géométrie. Le méso-espace fera référence dans le texte à l'espace associé à une cour d'établissement scolaire et le micro-espace à une feuille ou un ouvrage.

1 Van den Heuvel-Panhuizen, "The didactical use of models in Realistic Mathematics Education...." ".

${ }^{2}$ Berthelot \& Salin, L'enseignement de l'espace et de la géométrie dans la scolarité obligatoire.

R. B. E. C. T., vol 6, núm. 1, jan-abr.2013 ISSN - 1982-873X

Artigo apresentado na Premières Journées d'études organisées à Lyon (France) 


\section{Illustration par un exemple d'un problème en situation}

Dans le cadre d'une liaison possible entre les mathématiques et les méthodes d'utilisation de topomètres électroniques enseignées au sein de l'ENTE, nous proposons de revisiter la trigonométrie par un problème professionnel de mesure de distances inaccessibles dans le mésoespace. Ce type de problématique, n'est certes pas nouveau à ce détail près qu'au cours de I'histoire, les situations proposées, en particulier par Clairaut (1741), étaient représentées directement dans les ouvrages c'est-à-dire dans le micro-espace. Aussi, nous prenons en compte :

- la possibilité de mesurer de façon effective des distances inaccessibles dans le mésoespace.

- la possibilité d'évoquer cette action à partir de la représentation des situations de ce domaine dans le micro-espace. Notre idée est que la modélisation qui assurerait précisément le passage du méso-espace au micro-espace contribuerait à la construction du sens d'une notion mathématique pour les étudiants.

\subsection{Le problème général}

Au cours de la première séance, certaines règles de construction sont rappelées aux étudiants. En France, les bâtiments doivent respecter des règlements édictés en particulier par les Plans Locaux d'Urbanisme. L'une de ces règles exige qu'une personne doit pouvoir apercevoir même partiellement un édifice classé Monument Historique de tout lieu situé sur un cercle de rayon 500 mètres et dont le centre est à l'intérieur du bâtiment classé. Autrement dit, aucun immeuble ne doit obstruer totalement la vue du bâtiment classé dans un rayon de 500 mètres. Les élèves sont ensuite confrontés à la situation suivante. Un immeuble de 9 mètres de hauteur doit s'implanter à moins de 500 mètres d'un édifice classé Bâtiment de France dont on ne connaît pas la hauteur. Les étudiants doivent représenter à l'échelle la zone interdite d'implantation de la construction. Ils sont amenés à comprendre qu'ils doivent avant tout trouver une méthode pour mesurer sur le terrain la hauteur de l'édifice classé.

\subsection{Situations de visée dans le méso-espace}

Un travail, que nous ne détaillons pas ici, préalable à l'activité qui suit, favorise l'émergence chez les étudiants des conditions correctes de visée avec une lorgnette en particulier le fait qu'il faut être de face donc perpendiculaire au tableau sur lequel est fixée la mire. Quinze de ces instruments sont construits à partir de boîtes parallélépipédiques différentes. Chacun est percé d'un trou de visée et d'une fente qui sont centrés. En binômes et dans la cour, les étudiants visent avec ces lorgnettes une mire composée d'une bande de papier de 1,50 mètre de long et de 15 centimètres de large fixée horizontalement sur un tableau. Pour cela, ils font coïncider les 
milieux de la fente et de la mire ainsi que leurs extrémités. Afin de faciliter cette visée, le milieu et les extrémités de la mire sont représentés par troistraits et le milieu de la fente d'une lorgnette est matérialisé par un morceau de ficelle. Chaque binôme marque le lieu de visée de sa lorgnette sur le sol à l'aide de cartons d'identification numérotés de 1 à 15 . Pour ces derniers, les choix de leur taille et leur rassemblement in fine dans trois espaces relativement restreints entraînent leur superposition au moins partielle.

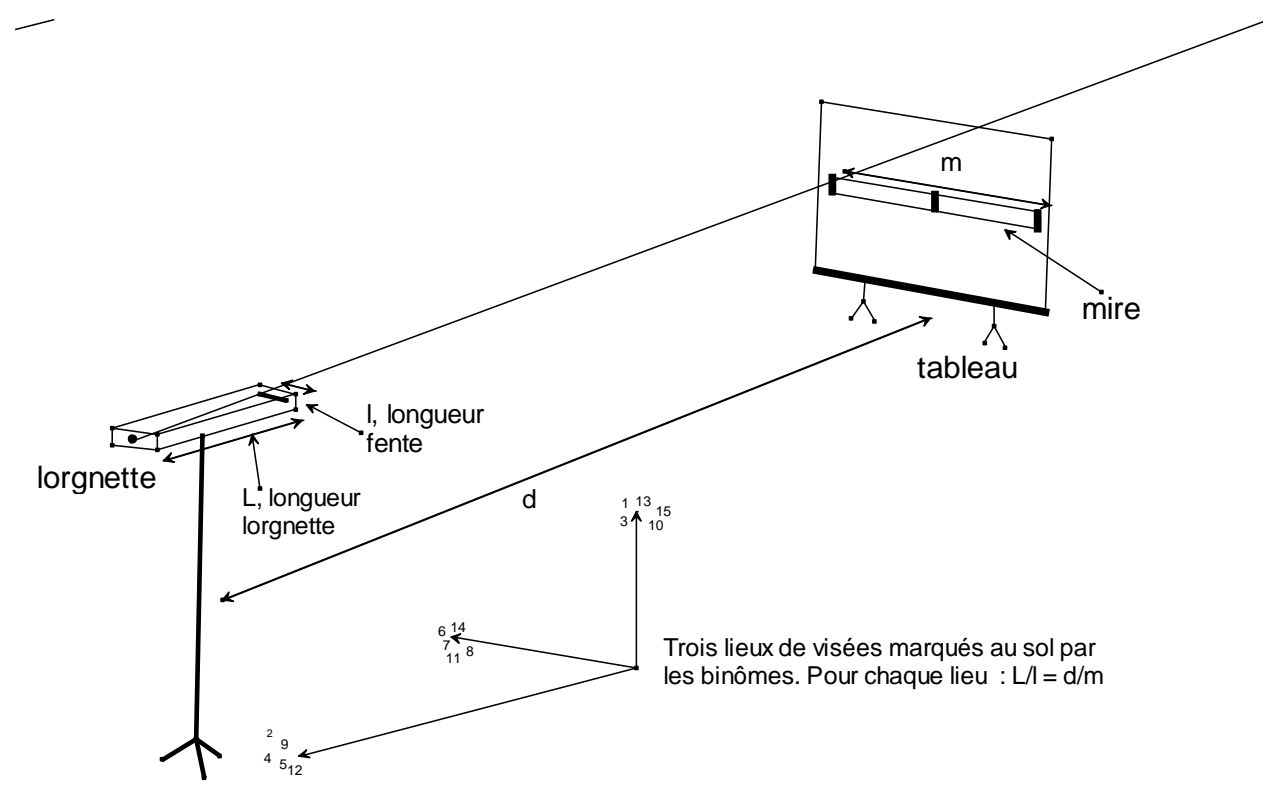

Nous nous trouvons au niveau du modèle analogique de Paul Ricoeur pour lequel, par exemple, il est possible de penser que par analogie cette situation des lorgnettes fonctionne de la même façon que le phénomène d'éclipse totale du soleil.

\subsection{Situation de modélisation de la visée et anticipation dans le micro-espace}

Le but final de cette deuxième séance est de savoir, grâce à une première démarche et sans se rendre dans la cour, si une mire pourrait être vue à l'aide d'une lorgnette dont une matérialisation dans le plan est mise à disposition des élèves.

Pour faire face à cette contrainte plus forte, une représentation de la situation est nécessaire. Représenter des lorgnettes utilisées dans l'espace à trois dimensions par un dessin plan constitue une première modélisation à l'échelle au sens de Ricoeur qui est loin d'être évidente comme nous avons pu le constater. Plus précisément, l'objectif pour les étudiants est d'aboutir, en premier lieu, à une représentation à l'échelle des lorgnettes par des rectangles en papier calque sur lesquels sont indiquées la fente et le trou de visée puis, en second lieu, à une représentation de la situation de visée. Cette dernière permet, d'une part, la mise en évidence de 
l'équivalence des lorgnettes à partir de la superposition de leur calque et de la matérialisation des champs de visée et, d'autre part, d'aboutir à la première procédure d'anticipation d'une visée dans le micro-espace. Une deuxième difficulté est liée au fait que des trajets lumineux immatériels doivent être tracés.

Il s'agit, dans le cadre de la modélisation proposée, de produire un objet spatio-graphique pour lequel les imperfections de tracé sont également fondamentales.

Une phase collective est tout d'abord mise en place en ce qui concerne la schématisation qui doit permettre aux étudiants d'imaginer une façon de représenter la lorgnette et la visée ainsi que les longueurs qui sont retenues pour la modélisation ultérieure. En confrontant leur représentations liées aux trois catégories ci-dessous, les étudiants se mettent d'accord sur l'une d'entre elles c'est-à-dire celle qui permet de matérialiser les lorgnettes par des rectangles faisant apparaître la fente et le trou de visée. Ce dessin va à l'encontre des schémas dans l'espace qu'un certain nombre d'entre eux produit. En effet, quelques uns sont tentés de schématiser la visée dans l'espace à trois dimensions. D'autres produisent des schémas hybrides relevant à la fois du plan et de l'espace. Mais pour la plupart, la représentation rectangulaire plane est proposée assez rapidement. Après échanges mutuels, un schéma qui permet de retenir l'essentiel est alors choisi par le groupe pour ensuite passer à la phase de modélisation.

S'en suit un travail en binôme pour la mise au point définitive de la représentation des lorgnettes à l'échelle, dont le rapport est imposé à l'ensemble des groupes, tenant compte du centrage de la fente et du trou de visée. Chaque binôme reproduit sa lorgnette en trois exemplaires.

Une fois ce travail effectué, neuf groupes sont constitués. Chaque groupe reçoit tous les calques d'un des trois ensembles de lorgnettes équivalentes afin de percevoir les champs de visées identiques par superposition puis pour anticiper une visée du micro-espace.

La problématique pratique intervient lorsque les étudiants manipulent les lorgnettes pour en retenir des informations et lorsqu'ils exécutent effectivement les représentations. Mais la problématique de modélisation prend déjà le relais lorsqu'il s'agit d'anticiper sur les réponses qui pourraient être encore obtenues par la visée, mais dont l'enseignant exige qu'elles soient trouvées dans le système symbolique du modèle. Les objets sont représentés mais pas encore au sein de la géométrie ; ils sont encore dans l'espace physique et sont manipulés de façon pratique. Les validations sont perceptives.

Les calques sont correctement positionnés les uns sur les autres pour signifier, après avoir tracé leur champ de visée, que les lorgnettes d'un même tas sont équivalentes. L'anticipation d'une visée est évoquée et exécutée par les étudiants. Ils ont globalement bien assimilé le fait que, pour qu'une mire soit perçue correctement à l'aide d'une lorgnette dans le micro-espace, il 
faut d'une part, que les milieux de la mire, de la fente et le trou de visée soient alignés et que, d'autre part, cette mire entre exactement dans le champ de visée de la lorgnette.

\subsection{Situations expliquant l'équivalence des lorgnettes et anticipant une visée par une nouvelle méthode : le calcul}

L'objectif de cette séance est d'émettre une conjecture pour expliquer l'équivalence des lorgnettes qui ont le même champ de visée en mettant en évidence la proportionnalité de leur longueur et de leur fente. Pour cela, sont consignées au tableau et pour chaque lorgnette, les mesures des longueurs de la fente, de la longueur et de la largeur de la lorgnette et de la mire ainsi que les distances dans le méso-espace des trois lieux de visée à la mire. Les contraintes liées à cette situation sont fortes en particulier celle de faire émerger une propriété de proportionnalité à partir de mesures prises dans la réalité car ces dernières sont alors entachées d'approximations. C'est pour cette raison que les caractéristiques des lorgnettes d'un des trois groupes sont telles que le rapport peut être facilement identifié (largeur fente/longueur lorgnette = 1/10), ce que les étudiants font. Ils déduisent une partie de la généralisation attendue : le rapport longueur fente/longueur lorgnette est à peu près le même pour les lorgnettes d'un même tas. Ils finissent par reconnaître la tangente qu'ils ont déjà abordée il y a longtemps. Ils trouvent qu'à chaque fois le rapport précédent est également celui de la longueur de la mire sur distance lorgnette-mire. Ce constat doit permettre d'anticiper, par le calcul, une visée du méso-espace. Même si une phase calculatoire prend place, les validations sont perceptives. Mais un discours prenant appui sur des formulations discursives commence à apparaître. La problématique géométrique prend place lors de la phase de démonstration de l'indépendance de la tangente dans un triangle rectangle fondée sur le théorème de Thalès et donc sur les nombres réels. Nous nous trouvons au niveau de la géométrie au sein de laquelle les objets sont définis par une formulation discursive.

\subsection{Retour dans le méso-espace}

La situation de réinvestissement, qui est un cas d'école permettant de comprendre la situation, a pour but d'apporter une solution pratique au problème de mesure de la hauteur du Bâtiment de France dont il est question au début, grâce à l'utilisation d'un télémètre en bois présenté ${ }^{1}$. Après avoir étudié les mathématiques, Errard de Bar-le-Duc a été formé par des ingénieurs italiens qui étaient au service du Duc de Lorraine dans le but de concevoir des fortifications. Mais il publia également des ouvrages de mathématiques qui ont été inspirés par ces pratiques professionnelles et dans lesquels les instruments avaient une grande place. Ainsi, le

${ }^{1}$ Errard de Bar-le-Duc, 1594

R. B. E. C. T., vol 6, núm. 1, jan-abr.2013 ISSN - 1982-873X 
télémètre en bois se compose de deux règles graduées en centimètres l'une verticale qui peut glisser le long d'une autre Horizontale (fig.3). Une troisième branche est articulée avec cette dernière. L'utilisateur doit viser perpendiculairement le

bâtiment classé avec la règle horizontale et faire coïncider le sommet de l'édifice avec l'extrémité de la troisième branche articulée. Pour déterminer la distance inconnue, il mesure la distance qui le sépare de l'édifice, par exemple 20 mètres, et il place la règle verticale sur la graduation 20 de la règle horizontale. Le nombre de centimètres qui apparaît à l'intersection de la règle verticale et de la partie articulée donne la hauteur réelle cherchée moyennant une conversion et la prise en compte de la taille de l'utilisateur. II y a

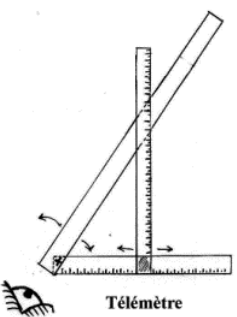

Figure 3 proportionnalité entre les longueurs du télémètre et les longueurs réelles. Pour résoudre

complètement le problème initial, les étudiants calculent la distance maximale qui sépare l'édifice classé du projet de construction grâce à la tangente. La solution consiste alors à tracer à l'échelle un anneau plan défini par l'intersection d'un disque de rayon $500 \mathrm{~m}$ et du disque de même centre dont le rayon est la longueur précédente.

De nos jours, les techniciens n'utilisent plus de télémètre en bois. Nous nous trouvons à la transition entre le domaine de la légitimation de l'utilisation d'un instrument rudimentaire qui répond de façon pratique au problème de mesure de distances inaccessibles et l'emploi d'instruments de topographies électroniques contemporains comme le tachéomètre. La mise en station d'un tachéomètre s'effectue à l'aide d'un trépied et à la verticale exacte d'un point connu en coordonnées $\mathrm{NGF}^{1}$ (trottoir, bouche d'égout etc.) à l'aide d'un fil à plomb et d'un niveau à bulle sphérique. Sa base est parfaitement horizontale grâce à l'utilisation d'une nivelle torique et d'une nivelle sphérique. Une lunette qui peut pivoter verticalement permet de viser le sommet du Bâtiment de France dont on cherche la hauteur. L'instrument communique alors l'angle de visée. La distance séparant le tachéomètre du bâtiment se mesure au laser à l'aide d'un prisme réflecteur tétraédrique placé à la verticale du point dont on souhaite mesurer la hauteur en l'occurrence le sommet du Monument Historique. Connaissant l'angle de visée et la distance à l'immeuble, une simple application de la tangente dans un triangle rectangle permet de calculer cette hauteur. Un niveau automatique communique l'altitude du pied du bâtiment ce qui permet éventuellement de rectifier le précédent résultat.

\footnotetext{
${ }^{1}$ Nivellement Général de la France
} 


\section{Qu'est-ce que modéliser?}

Nous proposons à présent de peaufiner notre définition de la modélisation du réel par une approche sémiotique de ce concept afin de repérer les différentes étapes du passage de la " réalité » vers le monde des mathématiques. Nous envisageons la réalité en général en termes de contexte et d'événement. Le contexte se décompose selon le binôme matériel/action. Dans notre étude, le contexte initial est la visée (action) d'une mire avec des lorgnettes (matériel) dans le méso-espace (environnement). Au départ, le contexte est détaché de tout constat de fait. Par événement, nous comprenons la donnée d'un lieu, d'un instant et d'interprétations de faits en rapport avec un contexte initial. La modélisation du réel concerne tous les différents mouvements établis entre, d'une part, un contexte et un événement qui lui est rattaché qui sont perçus et constatés dans le langage courant et, d'autre part, les diverses représentations de ce contexte et de cet événement engendrant de nouvelles interprétations à leur sujet ou générant de nouveaux contextes/événements. Modéliser le contexte initial consiste à modéliser les objets, le matériel et I'action. Nous construisons une première acception de la " modélisation » qui est plutôt réaliste ou informelle au cours de laquelle, à chaque étape, le contexte et l'événement initiaux sont apparents de façon évidente ou latente. Ce niveau de modélisation réaliste génère des modèles se référant au contexte initial permettant, d'une part, l'émergence de nouvelles interprétations de ce contexte et des événements qui lui sont rattachés et, d'autre part, la naissance de nouveaux événements dans lesquels les mathématiques peuvent déjà intervenir. Le contexte et l'événement initiaux peuvent faire l'objet d'une modélisation réaliste perceptible ou d'une modélisation réaliste imaginable. Le second sens est lié à la modélisation formelle obtenue à l'aide de modèles ne se référant plus au contexte initial et pour laquelle les événements et les objets, détachés de toute référence à la situation de départ, sont strictement mathématiques. Dans les étapes de modélisations liées à un fait tiré du réel, suivant l'idée qu'un modèle possèdera toutes ces composantes directement perceptibles dans la réalité de départ ou seulement imaginables ou que ce modèle se traduira par des expressions mathématiques, nous

distinguons respectivement les interprétations "perceptive », "imaginable " ou «mathématique » d'un fait, ce qui nous conduit à définir les notions d'événement "perceptible », d'événement "imaginable » ou d'événement "mathématique » liées à un contexte.

\subsection{Avant le début de la modélisation}

Contexte initial : dans la cour de l'établissement, les binômes visent à l'aide de lorgnettes une mire fixée sur un tableau en faisant coïncider les extrémités et le milieu de la mire avec les extrémités et le milieu de la fente de la lorgnette.

Evénement " perceptible » initial : dans la cour, (le lieu), après que tous les binômes ont procédé à la visée en ayant marqué l'emplacement de cette dernière (l'instant), certains élèves R. B. E. C. T., vol 6, núm. 1, jan-abr.2013 ISSN - 1982-873X 
remarquent que les cartons identificateurs sont regroupés en trois tas ( $1^{\text {ère }}$ interprétation " perceptive " du fait : équivalence des lorgnettes) et d'autres rajoutent que des lorgnettes différentes, sans préciser en quoi elles le sont, peuvent avoir le même lieu de visée $\left(2{ }^{\text {ème }}\right.$ interprétation « perceptive » du fait : équivalence des lorgnettes).

Evénement "mathématique » initial :dans la cour, (le lieu), après que tous les binômes ont procédé à la visée en ayant marqué l'emplacement de cette dernière (l'instant), certains élèves auraient pu dire que des lorgnettes de dimensions distinctes peuvent malgré tout avoir le même lieu de visée ( $1^{\text {ère }}$ interprétation « mathématique » du fait : équivalence des lorgnettes), ce qui n'a pas été le cas.

\subsection{Les différentes étapes de la modélisation:}

La modélisation du contexte initial passe par la modélisation des objets, du matériel et des actions initiales menées à l'aide de ces supports.

Modèle perceptible du contexte initial (matériel) : dans la salle organisée en ateliers, les apprenants représentent les lorgnettes par des rectangles à l'échelle sur papier calque avec la fente et le trou de visée bien centrés. II s'agit d'une modélisation perceptible du matériel lié au contexte initial car nous modélisons une chose effectivement visible dans le monde palpable. En effet, des rectangles de même type percés d'un point de visée et fendus d'un segment représentant les lorgnettes dans le plan peuvent aussi apparaître dans le contexte matériel initial si nous procédons à une découpe en long des lorgnettes parallélépipédiques suivant le plan défini par le trou et la fente de visée.

Modèle imaginable du contexte initial (matériel) : pour pouvoir matérialiser la visée dans le plan, les élèves tracent le champ de visée de leur lorgnette. Le modèle du matériel en rapport avec le contexte initial est ici réaliste imaginable car les champs sont réels mais impalpables dans le contexte initial.

Modèle imaginable du contexte initial (action) : après avoir modélisé une lorgnette et son champ de visée, l'action de visée d'une mire est modélisée. Dans le plan, les milieux de la mire et de la fente sont placés sur la bissectrice de l'angle de visée et leurs extrémités respectives sont situées sur les côtés de cet angle. C'est un premier exemple qui illustre le fait que la modélisation constitue une interface entre deux interprétations de la réalité. Ce rôle de médiation est apparent grâce au lien établi entre, d'une part, le fait qu'il fallait, pour viser correctement dans la cour, être de face et que les extrémités et les milieux des deux objets devaient coïncider et, d'autre part, l'idée que dans le modèle, les extrémités et les milieux en question se situent respectivement sur le bissectrice et les côtés de l'angle de visée. Ces trois modèles réalistes imaginables et perceptibles représentent une réalité.

Evénement "concret perceptible » dans la nouvelle réalité : le modèle imaginable du 
contexte initial correspond à une nouvelle réalité et donne lieu à l'apparition d'un nouvel événement concret perceptible : dans la salle de classe (lieu), après avoir représenté les lorgnettes à l'échelle sur papier calque (instant), les élèves constatent par superposition des calques la coïncidence de cinq angles.

Modélisation de l'événement initial.

Modèle imaginable de l'événement initial : l'événement concret perceptible précédent peut aussi être interprété comme étant un événement "concret imaginable » dans le modèle lié à l'événement initial : dans la salle de classe (lieu), après avoir représenté les lorgnettes à l'échelle sur papier calque (instant), les élèves constatent que l'équivalence des lorgnettes correspond au fait qu'elles ont le même champ de visée. (1 ${ }^{\text {ère }}$ interprétation « imaginable » dans le modèle du fait " équivalence des lorgnettes »). La modélisation est une interface entre un événement lié au modèle et un autre en rapport avec le contexte initial.

Evénement "concret mathématique " dans le modèle lié à l'événement initial :un événement " mathématique " peut être rattaché à une interprétation mathématique d'un fait perceptible: dans la salle de classe (le lieu), après avoir énoncé que les champs de visée sont équivalents (l'instant), des élèves disent : "Les lorgnettes équivalentes ont des angles de visée égaux " ( $2^{\text {ème }}$ interprétation mathématique d'un fait perceptible). Ici aussi nous pouvons dire que la modélisation est une interface entre différents types d'interprétations de la réalité car un retour au contexte initial est important si nous voulons que l'idée de l'équivalence de deux lorgnettes en termes de champ de visée soit dépassée. En quoi les lorgnettes sont-elles différentes? Elles n'ont pas les mêmes mesures de longueurs. D'où l'apparition d'un nouvel événement "concret mathématique " dans le modèle lié à l'événement initial : dans la salle de classe (le lieu), après avoir énoncé que les champs de visée sont équivalents (l'instant), des élèves disent : "Les lorgnettes équivalentes ont certaines mesures de longueurs proportionnelles» ( 3 ème interprétation mathématique d'un fait perceptible). Une fois cette étape franchie, nous poursuivons la modélisation du contexte et d'événements initiaux mais dans le cadre strict des mathématiques puisqu'il s'agit alors de démontrer une conjecture. Cela qui relève de la seconde acception du terme « modélisation ».

Ce second sens est la modélisation formelle obtenue à l'aide de modèles ne se référant plus au contexte initial et pour laquelle les événements, détachés de toute référence à la situation de départ, sont strictement mathématiques. II s'agit de démonter que la tangente d'un angle donné est indépendante de triangle rectangle dans lequel il serait représenté. Cette démonstration ne peut pas réellement être faite au Lycée puisqu'elle nécessite l'apport des nombres réels qui ne sont pas construits à ce niveau. Cette indépendance est bien sûr admise dès le collège. 


\section{Conclusion}

Les différentes étapes de la modélisation qui ont été mise en évidence dans le cadre des études topographiques menées au sein de l'ENTE n'ont pas pour objectif de résoudre des problèmes spécifiques de cet enseignement mais plutôt de montrer que ce questionnement reliant la réalité matérielle aux mathématiques peut trouver un écho intéressant en termes d'objectifs d'apprentissage dans l'enseignement post baccalauréat technique. L'articulation entre une discipline technique, la topographie, et une discipline générale, les mathématiques, semble pouvoir se mettre en place à partir de cette problématique de modélisation. Les deux types de modélisations formelles et informelles participent de la modélisation mathématique dans l'enseignement qui relève de la dialectique établie entre les différentes étapes de modélisation d'un contexte et d'événements initiaux.

Notre situation met en évidence l'idée que la modélisation est une interface entre différents types d'interprétations de la réalité. Elle nous montre en l'occurrence qu'il est possible de procéder à une modélisation d'un problème tiré de la réalité matérielle par des allers et retours entre un événement constaté dans un contexte initial et un événement interprété dans un modèle qui correspond à une nouvelle réalité. Mais finalement quel est l'intérêt de proposer un tel enseignement des mathématiques ? A partir d'une situation tirée de la réalité matérielle, les différents modèles qui représentent autant de niveaux distincts d'interprétation et de formalisation de cette réalité peuvent favoriser la mise en place d'une mathématisation " horizontale " du monde qui part du monde " palpable » pour arriver au monde des symboles et d'une mathématisation "verticale " qui naît et évolue principalement dans le monde des symboles, ces deux types de mathématisation étant liés à ce que nous appelons " composante interne " et "composante externe " du sens des mathématiques. Dans le cadre de notre situation, la géométrie enseignée apparaît comme un langage permettant, d'une part, de décrire et d'interpréter la réalité et, d'autre part, de construire et de modéliser l'espace physique. Ces deux fonctions de la géométrie en situation d'enseignement sont importantes en ce qui concerne la manière dont des étudiants sont susceptibles de s'approprier un savoir géométrique. La question qui demeure encore dans le cadre de cette situation d'enseignement serait de comprendre la manière dont le contexte interne de l'étudiant pourrait être mieux pris en compte afin que, par exemple, le passage du constat de l'égalité des angles de visée à l'égalité de rapports de mesures de longueurs soit le plus à la charge possible des étudiants. 


\section{Bibliographie}

Bartolini Bussi. Maria. \& Boero, Paolo, «Teaching and Learning Geometry in Contexts, Perspectives on the teaching of geometry for the 21st century ", In ICMI study, Dordrecht, NL, Ed. Kluwer, 1998, pp. 52-62.

Berthelot René \& Salin Marie Hélène, L'enseignement de l'espace et de la géométrie dans la scolarité obligatoire, Thèse de doctorat. Université Bordeaux I. LADIST, 1992.

Chevallard, Yves, La transposition didactique, Du savoir savant au savoir enseigné, Grenoble,La Pensée Sauvage, 1991.

Clairaut, A, Eléments de géométrie, Paris, Hachette, 1741.

Dapueto, Carlo, \& Parenti, Laura, "Contributions and obstacles of contexts in the developpement of mathematical knowledge. Contributions and obstacles of contexts in the development of mathematical knowledge ». Educational Studies in Mathematics, 39, 1999, pp. 1-21.

Douady, Régine, "Jeux de cadres et dialectique outil-objet", Recherche en Didactique des Mathématiques Vol 7.2, Grenoble, La Pensée Sauvage, 1986.

Douek, Nadia, « Argumentation and conceptualization in context : a case study on sunshadows in primary school ». Educational Studies in Mathematics, 39, 1999, pp. 89 -110.

Errard de Bar-le-Duc, Jean, La géométrie et pratique d'icelle, Paris, Michel Daniel, 1594.

Gravemeijer, Koeno, \& Doorman, Michiel, « Context problems in realistic mathematics education : a calculus course as an example », Educational Studies in Mathematics, 39, 1999, pp. 111-129.

Ricoeur, Paul, De l'interprétation. Essai sur Freud, Paris, Seuil, 1965.

Thirion, Maurice, Les mathématiques et le réel, Paris, Ellipse, IREM - Histoire des mathématiques, 1999.

Trigueros, M, « La modélisation de situations réelles et l'utilisation d'une théorie de construction de la connaissance dans l'enseignement des mathématiques universitaires", Actes Colloque DIDIREM Université Paris Diderot Paris 7, 2008.

Van Den Heuvel-Panhuizen, Marja, "The didactical use of models in Realistic Mathematics Education : An example from longitudinal trajectory on percentage ", Educational Studies in Mathematics 54, 2003, pp. 9-35.

Vygotski, Lev Semionovitch, Pensée et langage, traduction F Sève, Paris, La Dispute, (1934), 1984. 